Bull. Korean Math. Soc. 48 (2011), No. 3, pp. 555-574

DOI 10.4134/BKMS.2011.48.3.555

\title{
GLOBAL STABILITY OF THE VIRAL DYNAMICS WITH CROWLEY-MARTIN FUNCTIONAL RESPONSE
}

\author{
Xueyong Zhou and Jingan Cui
}

\begin{abstract}
It is well known that the mathematical models provide very important information for the research of human immunodeciency virus type. However, the infection rate of almost all mathematical models is linear. The linearity shows the simple interaction between the T-cells and the viral particles. In this paper, a differential equation model of $\mathrm{HIV}$ infection of $\mathrm{CD} 4^{+} \mathrm{T}$-cells with Crowley-Martin function response is studied. We prove that if the basic reproduction number $R_{0}<1$, the HIV infection is cleared from the T-cell population and the disease dies out; if $R_{0}>1$, the HIV infection persists in the host. We find that the chronic disease steady state is globally asymptotically stable if $R_{0}>1$. Numerical simulations are presented to illustrate the results.
\end{abstract}

\section{Introduction}

Human immunodeficiency virus (HIV) is a lentivirus, a member of the retrovirus family, that causes acquired immunodeficiency syndrome (AIDS), a condition in humans in which the immune system begins to fail, leading to lifethreatening opportunistic infections. HIV transmission requires contact with body fluids containing infected cells or plasma. HIV may be present in any fluid or exudate that contains plasma or lymphocytes, specifically blood, semen, vaginal secretions, breast milk, saliva, or wound exudates. Although theoretically possible, transmission by saliva or droplet nuclei produced by coughing or sneezing is extremely rare, if it occurs. HIV is not transmitted by casual contact or even by the close nonsexual contact that occurs at work, school, or home. The most common means of transmission is direct transfer of bodily fluids either through sharing contaminated needles or sexual relations.

Received September 18, 2009; Revised February 20, 2010.

2010 Mathematics Subject Classification. 34D20, 92D30.

Key words and phrases. HIV infection, permanence, globally asymptotical stability.

This work is supported by the National Natural Science Foundation of China (No. 10771104 and No. 10771179), program for Innovative Research Team (in Science and Technology) in University of Henan Province and Innovation Scientists and Technicians Troop Construction Projects of Henan Province, Program for Key Laboratory of Simulation and Control for Population Ecology in Xinyang Normal University (No. 201004) and Natural Science Foundation of the Education Department of Henan Province (No. 2011B110028). 
The HIV infection is an infectious disease characterized by continual decrease of $\mathrm{CD}^{+}$lymphocyte cells, invariably causing the death of the host. The main target is $\mathrm{CD} 4^{+} \mathrm{T}$ helper cell; a type of $\mathrm{T}$ cell. $\mathrm{T}$ cells are an important part of the immune system because they help to facilitate the body's response to many common but potentially fatal infections. Without enough T-cells the body's immune system is unable to defend itself against many infections. In a normal person, the level of $\mathrm{CD}^{+} \mathrm{T}$ cells in the peripheral blood is regulated at a level between 800 and $1200 \mathrm{~mm}^{-3}$. By ways that are not yet completely understood, HIV's life cycle directly or indirectly causes a reduction in the number of T-cells in the body, eventually resulting in an increased risk of infections. Over time, there are not enough T-cells to defend the body. At this stage, a person is said to have Acquired Immunodeficiency Syndrome, or AIDS, and becomes susceptible to infections that a healthy immune system could deal with. The time in between the first infection and initiation of antibody synthesis is usually 6-12 weeks. The median time to receive an AIDS diagnosis among those infected with HIV is 7-10 years.

Mathematical modelling has been proven to be valuable in understanding the dynamics of HIV. And Several mathematical models have been proposed to describe the in vivo dynamics of $\mathrm{T}$ cell and HIV interaction (see, for example, $[1,12,25,26])$. This is because HIV mathematical models can provide insights into the dynamics of viral load in vivo. And they may pay a significant role in the development of a better understanding of HIV and drug therapies.

The basic mathematical model of HIV pathogenesis in-host describes interactions of the immune system and the virus by including healthy and infected $\mathrm{CD}^{+}{ }^{+}$T-cells and HIV virions $[10,9,11,14,23]$. A classic model for HIV dynamics was proposed by Perelson et al. in $[18,19]$ as follows:

$$
\left\{\begin{array}{l}
\frac{d T}{d t}=s-d T-\beta T V \\
\frac{d I}{d t}=\beta T V-\delta I \\
\frac{d V}{d t}=p I-c V
\end{array}\right.
$$

The first equation of (1.1) represents the dynamics of the concentration of healthy $\mathrm{CD}^{+}$cells $(T) ; s$ represents the rate (assumed constant) at which new $\mathrm{CD} 4^{+}$T-cells are generated. The death rate of healthy cells is $d$. In the case of active HIV infection the concentration of healthy cells decreases proportionally to the product $\beta T V$, where $\beta$ represents a coefficient that depends on various factors, including the velocity of penetration of virus into cells and the frequency of encounters between uninfected cells and free virus. The second equation of (1.1) describes the dynamics of the concentration of infected CD4 ${ }^{+}$ cells $(I) ; \beta$ is the rate of infection; $\delta$ is the death rate of infected cells. The third equation of (1.1) describes the concentration of free virions $(V)$, which 
are produced by the infected cells at a rate $p$, and $c$ is the death rate of the virions.

Usually the rate of infection in most HIV models is assumed to be bilinear in the virus $V$ and the uninfected $\mathrm{CD}^{+}$cells $T$. However, the actual incidence rate is probably not linear over the entire range of $V$ and $T$. Thus, it is reasonable as to assume that the infection rate of modelling HIV infection in Crowley-Martin function response, $\frac{\beta T V}{1+a T+b V+a b T V}$, where $a, b \geq 0$ are constants. The Crowley-Martin type of functional response was introduced by $\mathrm{P}$. H. Crowley and E. K. Martin [3]. When $a>0, b=0$, the Crowley-Martin type of functional response is simplified to Michaelis-Menten (or Holling type II) functional response. And when $a=0, b>0$, it expresses a saturation response. Moreover, when $a=0, b=0$, the Crowley-Martin type of functional response is simplified to a linear mass-action function response (or Holling type I functional response). In general we consider the model with Crowley-Martin functional response given by

$$
\left\{\begin{array}{l}
\frac{d T}{d t}=s-d T-\frac{\beta T V}{1+a T+b V+a b T V} \\
\frac{d I}{d t}=\frac{\beta T V}{1+a T+b V+a b T V}-\delta I \\
\frac{d V}{d t}=p I-c V .
\end{array}\right.
$$

The system (1.2) needs to be analyzed with the following initial conditions:

$$
T(0)>0, I(0)>0, V(0)>0 .
$$

We denote

$$
R_{+}^{3}=\left\{(T, I, V) \in R^{3}, T \geq 0, I \geq 0, V \geq 0\right\} .
$$

Standard and simple arguments show that the solutions of the system (1.2) exist and stay positive.

The rest of this article is organized as follows. In Section 2, we present some preliminaries. In Section 3, the local and global stability of disease-free equilibrium $E_{0}$ of the system (1.2) are studied. In Section 4, the permanence of the system (1.2) is investigated. Local and global asymptotic stability of the disease steady state $E^{*}$ are discussed in Section 5 . In Section 6 , the numerical simulations are presented to illustrate the results. Finally, the paper concludes with a brief discussion.

\section{Some preliminary results}

It is important to show positivity and boundedness for the system (1.2) as they represent populations. Positivity implies that populations surveries and boundedness may be interpreted as a natural restriction to growth as a consequence of limited resources. In this section, we present some basic results, such as the positive invariance of the system (1.2), the existence of equilibria, and the boundedness of solutions. 


\subsection{Positive invariance}

The model (1.2) can be written in the form

$$
\dot{X}(t)=G(X(t)),
$$

where $X(t)=\left(x_{1}, x_{2}, x_{3}\right)^{\top}:=(T, I, V)^{\top}, X(0)=(T(0), I(0), V(0))^{\top} \in R_{+}^{3}$ and

$$
G(X)=\left(\begin{array}{c}
G_{1}(X) \\
G_{2}(X) \\
G_{3}(X)
\end{array}\right)=\left(\begin{array}{c}
s-d T-\frac{\beta T V}{1+a T+b V+a b T V} \\
\frac{\beta T V}{1+a T+b V+a b T V}-\delta I \\
p I-c V
\end{array}\right) .
$$

It is easy to check that $\left.G_{i}(X)\right|_{x_{i}=0} \geq 0, i=1,2,3$. Due to the well known theorem by Nagumo [16], any solution of (1.2) with initial point $X_{0} \in R_{+}^{3}$, say $X(t)=X\left(t ; X_{0}\right)$, is such that $X(t) \in R_{+}^{3}$ for all $t>0$.

\subsection{Boundedness}

Theorem 2.1. There exists $M>0$ such that all the solutions satisfy $T(t)$, $I(t), V(t)<M$ for all large $t$.

Proof. Since all solutions of (1.2) are positive, by the first equation of (1.2) we have

$$
\frac{d T}{d t}=s-d T-\frac{\beta T V}{1+a T+b V+a b T V} \leq s-d T .
$$

Therefore, we have

$$
\frac{d T}{d t}<\frac{s}{d}+1
$$

for all large $t$, say $t>t_{0}$.

Set

$$
V_{1}(t)=T(t)+I(t) .
$$

Calculating the derivative of $V_{1}$ along the solutions of the system (1.2), we find

$$
\frac{d V_{1}(t)}{d t}=s-d T(t)-\delta I(t) \leq-h V_{1}(t)+s,
$$

where $h=\min (d, \delta)$. Recall that $T(t) \leq \frac{s}{d}+1$ for $t>t_{0}$. Then there exists $M_{1}$, depending only on the parameters of the system (1.2), such that $V_{1}(t) \leq M_{1}$ for $t>t_{0}$. Then $I(t)$ has an ultimately above bound. It follows from the third equation of the system (1.2) that $V(t)$ has an ultimately above bound, say, their maximum is an $M$. Then the assertion of Theorem 2.1 now follows and the proof is complete. This shows that the system (1.2) is dissipative.

Define

$$
\Omega=\left\{(T, I, V) \mid 0 \leq T \leq \frac{s}{d}+1,0 \leq I, V \leq M\right\} .
$$

Obviously, $\Omega$ is convex. 


\subsection{Equilibria}

Let $R_{0}=\frac{s p \beta}{\delta c(d+s a)}$. It is well-known the importance of the value, $R_{0}$, which is called as the basic reproductive ratio of the system (1.2). It represents the average number of secondary infection caused by a single infected $T$ cells in an entirely susceptible $T$ cells population throughout its infectious period. And it determines the dynamical properties of the system (1.2) over a long period of time.

It is easy to see that if $R_{0} \leq 1$, the disease-free steady state $E_{0}\left(T_{0}, 0,0\right)$ (where $T_{0}=\frac{s}{d}$ ) is the unique steady state, corresponding to the extinction of the free virus. The following theorem presents the existence and uniqueness of positive equilibrium if $R_{0}>1$.

Theorem 2.2. If $R_{0}>1$, then the system (1.2) has a unique interior equilibrium $E^{*}\left(T^{*}, I^{*}, V^{*}\right)$ (i.e., $\left.T^{*}>0, I^{*}>0, V^{*}>0\right)$, where $T^{*}, I^{*}$ and $V^{*}$ are given in the process of the proof.

Proof. From the system (1.2), such a point satisfies

$$
\left\{\begin{array}{l}
s-d T^{*}-\frac{\beta T^{*} V^{*}}{\beta T^{*} V^{*} T^{*}+b V^{*}+a b T^{*} V^{*}}=0, \\
\frac{1+a T^{*}+b V^{*}+a b T^{*} V^{*}}{1+\delta I^{*}=0} \\
p I^{*}-c V^{*}=0 .
\end{array}\right.
$$

We easily get

$$
a b p d T^{* 2}+(p \beta-\delta c a+b p d-a b s p) T^{*}-(\delta s+b p s)=0,
$$

and thus,

$$
T_{ \pm}^{*}=\frac{-(p \beta-\delta c a+b p d-a b s p) \pm \sqrt{(p \beta-\delta c a+b p d-a b s p)^{2}+4 a b p d(\delta s+b p s)}}{2 a b p d} .
$$

Moreover, simply algebraic computations show that $T_{+}^{*}>0$ and $T_{-}^{*}<0$. Therefore, system (1.2) possesses a unique interior equilibrium $E^{*}\left(T^{*}, I^{*}, V^{*}\right)$ given by

$$
\begin{aligned}
T^{*} & =\frac{-(p \beta-\delta c a+b p d-a b s p)+\sqrt{(p \beta-\delta c a+b p d-a b s p)^{2}+4 a b p d(\delta s+b p s)}}{2 a b p d}, \\
I^{*} & =\frac{1}{\delta}\left(s-d T^{*}\right), V^{*}=\frac{p}{c} I^{*}
\end{aligned}
$$

if an only if $s-d T^{*}>0$.

It is easily to see that if $R_{0}>1$, then $s-d T^{*}>0$. Hence, the system (1.2) has a unique interior equilibrium $E^{*}\left(T^{*}, I^{*}, V^{*}\right)$ if $R_{0}>1$. 


\section{Local and global stability of $E_{0}$}

Let $\widehat{E}(\widehat{T}, \widehat{I}, \widehat{V})$ be any arbitrary equilibrium. Then the characteristic equation about $\widehat{E}$ is given by

$$
\left|\begin{array}{ccc}
-d-\frac{\beta \widehat{V}+b \beta \widehat{V}^{2}}{(1+a \widehat{T}+b \widehat{V}+a b \widehat{T} \widehat{V})^{2}}-\lambda & 0 & -\frac{\beta \widehat{T}+a \beta \widehat{T}^{2}}{(1+a \widehat{T}+b \widehat{V}+a b \widehat{T} \hat{V})^{2}} \\
\frac{\beta \widehat{V}+b \beta \widehat{V}^{2}}{(1+a \widehat{T}+b \widehat{V}+a b \widehat{T} \widehat{V})^{2}} & -\delta-\lambda & \frac{\beta \widehat{T}+a \beta \widehat{T}^{2}}{(1+a \widehat{T}+b \widehat{V}+a b \widehat{T} \widehat{V})^{2}} \\
0 & p & -c-\lambda
\end{array}\right|=0 .
$$

For equilibrium $E_{0}\left(T_{0}, 0,0\right),(3.1)$ reduces to

$$
(\lambda+d)\left[\lambda^{2}+(c+\delta) \lambda+c \delta-\frac{p \beta T_{0}}{1+a T_{0}}\right]=0 .
$$

Hence, $E_{0}\left(T_{0}, 0,0\right)$ is locally asymptotically stable for $R_{0}<1$. And it is a saddle with $\operatorname{dim} W^{s}\left(E_{0}\right)=2, \operatorname{dim} W^{u}\left(E_{0}\right)=1$ for $R_{0}>1$. Then we have the following theorem.

Theorem 3.1. If $R_{0}<1, E_{0}\left(T_{0}, 0,0\right)$ is locally asymptotically stable; if $R_{0}>$ $1, E_{0}\left(T_{0}, 0,0\right)$ is unstable.

Theorem 3.2. If $R_{0}<1, E_{0}\left(T_{0}, 0,0\right)$ is globally asymptotically stable.

Proof. Define a Lyapunov function $L_{1}(T, I, V)$ as follows:

$$
L_{1}(T, I, V)=A_{1}\left(T-T_{0}-T_{0} \ln \frac{T}{T_{0}}\right)+I+\frac{\delta}{p} V,
$$

where $A_{1}>0$ is selected in the following proof. Calculating the time derivative of $L_{1}(T, I, V)$ along the positive solutions of the model (1.2), we obtain

$$
\begin{aligned}
\dot{L_{1}}= & A_{1}\left(\dot{T}-\frac{T_{0}}{T} \dot{T}\right)+\dot{I}+\frac{\delta}{p} \dot{V} \\
= & A_{1}\left(s-d T-\frac{\beta T V}{1+a T+b V+a b T V}-\frac{T_{0}}{T} s+d T_{0}+\frac{\beta T_{0} V}{1+a T+b V+a b T V}\right) \\
& +\frac{\beta T V}{1+a T+b V+a b T V}-\delta I+\frac{\delta}{p}(p I-c V) \\
= & d A_{1} T_{0}\left(2-\frac{T}{T_{0}}-\frac{T_{0}}{T}\right)+\frac{\beta T V}{1+a T+b V+a b T V}+\frac{A_{1} \beta T_{0} V}{1+a T+b V+a b T V} \\
& +\frac{A_{1} \beta T V}{1+a T+b V+a b T V}-\frac{\delta c}{p} V \\
= & d A_{1} T_{0}\left(2-\frac{T}{T_{0}}-\frac{T_{0}}{T}\right)+\frac{p \beta T V-p A_{1} \beta T V-\delta c a T V}{p(1+a T+b V+a b T V)}-\delta c a b T V^{2} .
\end{aligned}
$$


Select $A_{1}=\frac{\delta c}{p \beta T_{0}}$. Clearly, $L_{1}(T, I, V)$ is positive definite with respect to $\left(T-T_{0}, I, V\right)$. Furthermore, since

$$
\begin{aligned}
& 2-\frac{T}{T_{0}}-\frac{T_{0}}{T}<0,\left(T \neq T_{0}\right), \\
& 2-\frac{T}{T_{0}}-\frac{T_{0}}{T}=0,\left(T=T_{0}\right),
\end{aligned}
$$

and $R_{0} \leq 1$, we have that $\dot{L_{1}}(T, I, V) \leq 0$ for all $T, I, V>0$. Thus, the diseasefree steady state $E_{0}$ is stable. And $\dot{L_{1}}(T, I, V)=0$, when $T=T_{0}$ and $V=0$. Let $\Sigma_{0}$ be the largest invariant set in the set

$$
\Sigma=\left\{(T, I, V) \mid \dot{L_{1}}(T, I, V)=0\right\}=\left\{(T, I, V) \mid T=T_{0}, I \geq 0, V=0\right\} .
$$

We have from the third equation of (1.2) that $\Sigma_{0}=\left\{E_{0}\right\}$. It follows from LaSalle invariance principle that the disease-free steady state $E_{0}$ is globally asymptotically stable.

\section{The permanence of system (1.2)}

In this section, we shall present the permanence of the system (1.2).

Definition 4.1. The system (1.2) is said to be persistent if there are positive constants $m, M$ such that each positive solution $(T(t), I(t), V(t))$ of the system (1.2) with initial conditions (1.3) satisfies

$$
\begin{aligned}
& m \leq \lim _{t \rightarrow+\infty} \inf T(t) \leq \lim _{t \rightarrow+\infty} \sup T(t) \leq M, \\
& m \leq \lim _{t \rightarrow+\infty} \inf I(t) \leq \lim _{t \rightarrow+\infty} \sup I(t) \leq M, \\
& m \leq \lim _{t \rightarrow+\infty} \inf V(t) \leq \lim _{t \rightarrow+\infty} \sup V(t) \leq M .
\end{aligned}
$$

Definition 4.2 ([6, Metzler matrix $]$ ). Matrix $A$ is a Metzler matrix if and only if all its off-diagonal elements are non-negative.

Lemma 4.1 ([6, Perron-Frobenius Theorem $])$. Let $A$ be an irreducible Metzler matrix. Then, $\lambda_{M}$, the eigenvalue of $A$ of largest real part is real, and the elements of its associated eigenvector $v_{M}$ are positive. Moreover, any eigenvector of $A$ with non-negative elements belongs to span $v_{M}$.

In order to prove the permanence of system (1.2), we firstly present the following useful lemma. And we require the following compactness condition.

Condition 4.1. There exist $\epsilon>0$ and a subset $B$ of $X$ with the following properties:

(1) If $x \in X$ and $d\left(x, X_{2}\right)<\epsilon$, then $d\left(\Phi_{t}(X), B\right) \rightarrow 0$ as $t \rightarrow \infty$.

(2) The intersection $B \cup B_{\epsilon}\left(X_{2}\right)$ of $B$ with the $\epsilon$-neighborhood of $X_{2}$, $B_{\epsilon}\left(X_{2}\right)=\left\{x \in X ; d\left(x, X_{2}\right)<\epsilon\right\}$ has compact closure. 
Lemma 4.2 ([24]). Let $X_{1}$ be open in $X$ and forward invariant under $\Phi$. Further, let the compactness assumption (Condition 4.1) hold. Assume the $\Omega_{2}$,

$$
\Omega_{2}=\cup_{y \in Y_{2}} \omega(y), Y_{2}=\left\{x \in X_{2} ; \Phi_{t} \in X_{2}, \forall t>0\right\}
$$

has an acyclic isolated covering $M=\cup_{k=1}^{m} M_{k}$ such that each part $M_{k}$ of $M$ is a weak repeller of $X_{1}$. Then $X_{2}$ is a uniform strong repeller for $X_{1}$.

Theorem 4.1. If $R_{0}>1$, then the system (1.2) is permanent.

Proof. The result follows from an application of Lemma 4.2. Let us define $X_{1}$ be the interior of $R_{+}^{3}$ and $X_{2}$ be the boundary of $R_{+}^{3}$, i.e., $X_{1}=\operatorname{int}\left(R_{+}^{3}\right)$ and $X_{2}=\operatorname{bd}\left(R_{+}^{3}\right)$. This choice is in accordance with the conditions stated in this theorem. We begin by showing that sets $X_{1}$ and $X_{2}$ repel the positive solution of the system (1.2) uniformly. Furthermore, note that by virtue of Theorem 2.1, there exists a compact set $B$ in which all solutions of the system (1.2) initiated in $R_{+}^{3}$ ultimately enter and remain forever after. The compactness condition is easily verified for this set $B$. Denoting the $\omega$-limit set of the solution $x\left(t, x_{0}\right)$ of the system (1.2) starting in $x_{0} \in R_{+}^{3}$ by $\omega\left(x_{0}\right)$, we need to determine the following set:

$$
\Omega=\cup_{y \in Y_{2}} \omega(y) \text {, where } Y_{2}=\left\{x_{0} \in X_{2} \mid x\left(t, x_{0}\right) \in X_{2}, \forall t>0\right\} .
$$

From the system (1.2), it follows that all solutions starting in $\operatorname{bd}\left(R_{+}^{3}\right)$ but not on the $T$-axis leave $\mathrm{bd}\left(R_{+}^{3}\right)$ and that the $T$-axis is an invariant set, implying that $Y_{2}=\left\{(T, I, V)^{\top} \in \operatorname{bd}\left(R_{+}^{3}\right) \mid I=V=0\right\}$. Furthermore, it is easy to see that $\Omega=\left\{E_{0}\right\}$ as all solutions initiated on the $T$-axis converge to $E_{0}$. In fact, in the set $Y_{2}$, the system (1.2) becomes

$$
\dot{T}=s-d T .
$$

It is easy to see that $E_{0}$ is globally asymptotically stable if $R_{0}<1$. Hence, any solution $(T(t), I(t), V(t))$ of the system (1.2) initiating from $Y_{2}$ is such that $(T(t), I(t), V(t)) \rightarrow E_{0}\left(T_{0}, 0,0\right)$. Obviously, $E_{0}$ are isolated invariant, $\left\{E_{0}\right\}$ is isolated and is an acyclic covering. Next, we show that $W^{s}\left(E_{0}\right) \cap X_{1}=\varnothing$, i.e., $E_{0}$ is a weak repeller for $X_{1}$.

By definition, $E_{0}$ is a weak repeller for $X_{1}$ if for every solution starting in $x_{0} \in X_{1}$

$$
\lim _{t \rightarrow+\infty} d\left(x\left(t, x_{0}\right), E_{0}\right)>0 .
$$

We claim that (4.2) is satisfied if the following holds:

$$
W^{s}\left(E_{0}\right) \cap \operatorname{int}\left(R_{+}^{3}\right)=\varnothing .
$$

To see this, suppose (4.2) does not hold for some solution $x\left(t, x_{0}\right)$ starting in $x_{0} \in X_{1}$. In view of the fact that the closed positive orthant is positively invariant for system (1.2), it follows that $\lim _{t \rightarrow+\infty} d\left(x\left(t, x_{0}\right), E_{0}\right)=0$ and thus that $\lim _{t \rightarrow+\infty} x\left(t, x_{0}\right)=E_{0}$, which is clearly impossible if (4.3) holds. What 
remains to be shown is that (4.3) holds. The Jacobian matrix of the system (1.2) at $E_{0}$ is given in the following:

$$
J_{0}=\left(\begin{array}{ccc}
-d & 0 & -\frac{\beta T_{0}}{1+a T_{0}} \\
0 & -\delta & \frac{\beta T_{0}}{1+a T_{0}} \\
0 & p & -c
\end{array}\right)
$$

It easy to see that $J_{0}$ is unstable if $R_{0}>1$. In particular, $J_{0}$ possesses one eigenvalue with positive real part, which we denote as $\lambda_{+}$, and two eigenvalues with negative real part, $-d$, and an eigenvalue which we denote as $\lambda_{-}$. We proceed by determining the location of $E^{s}\left(E_{0}\right)$, the stable eigenspace of $E_{0}$. Clearly, $(1,0,0)^{\top}$ is an eigenvector of $J_{0}$ associated to $-d$. If $\lambda_{-} \neq-d$, then the eigenvector associated to $\lambda_{-}$has the following structure: $\left(0, p_{2}, p_{3}\right)^{\top}$, where $p_{2}, p_{3}$ satisfy the eigenvector equitation

$$
\left(\begin{array}{cc}
-\delta & \frac{\beta T_{0}}{1+a T_{0}} \\
p & -c
\end{array}\right)\left(\begin{array}{l}
p_{2} \\
p_{3}
\end{array}\right)=\lambda_{-}\left(\begin{array}{c}
p_{2} \\
p_{3}
\end{array}\right)
$$

If $\lambda_{-}=-d$, then $\lambda_{-}$is a repeated eigenvalue, and associated generalized eigenvector will possess the following structure: $\left(*, p_{2}, p_{3}\right)^{\top}$, where the value of $*$ is irrelevant for what follows and $p_{2}$ and $p_{3}$ also satisfy (4.4).

We claim that in both case, the vector $\left(p_{2}, p_{3}\right)^{\top} \notin R_{+}^{2}$. Obviously, the matrix in (4.4) is an irreducible Metzler matrix. From Definition 4.1, we know that it is a matrix with nonnegative off-diagonal entries. By using Lemma 4.1 (Perron-Frobenius Theorem), we get that the matrix in (4.4) possesses a simple real eigenvalue which is larger then the real part of any other eigenvalue, also called the dominant eigenvalue. Clearly, the dominant eigenvalue here is $\lambda_{+}$. But the Perron-Frobenius Theorem also implies that every eigenvector that is not associated with the dominant eigenvalue does not belong to the closed positive orthant. Applied here, this means that $\left(p_{2}, p_{3}\right)^{T} \notin R_{+}^{2}$. Consequently, $E^{s}\left(E_{0}\right) \cap \operatorname{int}\left(R_{+}^{3}\right)=\varnothing$, and therefore also $W^{s}\left(E_{0}\right) \cap \operatorname{int}\left(R_{+}^{3}\right)=\varnothing$, which concludes the proof.

\section{Local and global asymptotic stability of the disease steady state}

Firstly, we present sufficient conditions leading to locally asymptotically stable disease steady state.

Theorem 5.1. Suppose that

(i) $R_{0}>1$;

(ii) $c \delta\left(\frac{\beta V^{*}+b \beta V^{* 2}}{\left(1+a T^{*}+b V^{*}+a b T^{*} V^{*}\right)^{2}}+d\right)>\frac{d p\left(\beta T^{*}+b \beta T^{* 2}\right)}{\left(1+a T^{*}+b V^{*}+a b T^{*} V^{*}\right)^{2}}$;

(iii) $\begin{aligned} & \left(c+d+\delta+\frac{\beta V^{*}+b \beta V^{* 2}}{\left(1+a T^{*}+b V^{*}+a b T^{*} V^{*}\right)^{2}}\right)\left[c d+d \delta+(c+\delta) \frac{\beta V^{*}+b \beta V^{* 2}}{\left(1+a T^{*}+b V^{*}+a b T^{*} V^{*}\right)^{2}}\right] \\ & +(c+\delta) c \delta>p\left(c+\delta+2 d p+\frac{\beta V^{*}+b \beta V^{* 2}}{\left(1+a T^{*}+b V^{*}+a b T^{*} V^{*}\right)^{2}}\right) \frac{\beta T^{*}+b \beta T^{* 2}}{\left(1+a T^{*}+b V^{*}+a b T^{*} V^{*}\right)^{2}} .\end{aligned}$

Then the positive equilibrium $E^{*}\left(T^{*}, I^{*}, V^{*}\right)$ is locally asymptotically stable. 
Proof. For equilibrium $E^{*}\left(T^{*}, I^{*}, V^{*}\right),(3.1)$ reduces to

$$
\lambda^{3}+b_{1} \lambda^{2}+b_{2} \lambda+b_{3}=0
$$

where

$$
\begin{aligned}
& b_{1}=c+\delta+d+\frac{\beta V^{*}+b \beta V^{* 2}}{\left(1+a T^{*}+b V^{*}+a b T^{*} V^{*}\right)^{2}}>0, \\
& b_{2}=c(\delta+d)-\frac{p\left(\beta T^{*}+b \beta T^{* 2}\right)}{\left(1+a T^{*}+b V^{*}+a b T^{*} V^{*}\right)^{2}}+d \delta+\frac{(c+\delta)\left(\beta V^{*}+b \beta V^{* 2}\right)}{\left(1+a T^{*}+b V^{*}+a b T^{*} V^{*}\right)^{2}}, \\
& b_{3}=c \delta\left(\frac{\beta V^{*}+b \beta V^{* 2}}{\left(1+a T^{*}+b V^{*}+a b T^{*} V^{*}\right)^{2}}+d\right)-\frac{d p\left(\beta T^{*}+b \beta T^{* 2}\right)}{\left(1+a T^{*}+b V^{*}+a b T^{*} V^{*}\right)^{2}} .
\end{aligned}
$$

We also have

$$
\begin{aligned}
& b_{1} b_{2}-b_{3} \\
= & \left(c+d+\delta+\frac{\beta V^{*}+b \beta V^{* 2}}{\left(1+a T^{*}+b V^{*}+a b T^{*} V^{*}\right)^{2}}\right)\left[c d+d \delta+(c+\delta) \frac{\beta V^{*}+b \beta V^{* 2}}{\left(1+a T^{*}+b V^{*}+a b T^{*} V^{*}\right)^{2}}\right] \\
& +(c+\delta) c \delta-p\left(c+\delta+2 d p+\frac{\beta V^{*}+b \beta V^{* 2}}{\left(1+a T^{*}+b V^{*}+a b T^{*} V^{*}\right)^{2}}\right) \frac{\beta T^{*}+b \beta T^{* 2}}{\left(1+a T^{*}+b V^{*}+a b T^{*} V^{*}\right)^{2}} .
\end{aligned}
$$

By Routh-Hurwitz criterion [16], we have that $E^{*}\left(T^{*}, I^{*}, V^{*}\right)$ is locally asymptotically stable.

Next, we provide sufficient conditions leading to a globally asymptotically stable disease steady state if the conditions of the theorem are satisfied.

Firstly, we will summarize the main facts related to our research. Let us consider the system of differential equations

$$
\frac{d X}{d t}=F(X), X \in D,
$$

where $D$ is an open subset on $R^{3}$ and $F$ is twice continuously differentiable in $D$. The noncontinuable solution of (5.1) satisfying $X(0)=X_{0}$ is denoted by $X\left(t, X_{0}\right)$, the positive (negative) semi-orbit through $X_{0}$ is denoted by $\phi^{+}\left(X_{0}\right)$ $\left(\phi^{-}\left(X_{0}\right)\right)$, and the orbit through $X_{0}$ is denoted by $\phi(0)=\phi^{-}\left(X_{0}\right) \cup \phi^{+}\left(X_{0}\right)$. We use the notation $\omega\left(X_{0}\right)\left(\alpha\left(X_{0}\right)\right)$ for the positive (negative) limit set of $\phi^{+}\left(X_{0}\right)\left(\phi^{-}\left(X_{0}\right)\right)$, provided the latter semi-orbit has compact closure in $D$.

The system (5.1) is competitive in $D[7,21,22,27]$ if, for some diagonal matrix $H=\operatorname{diag}\left(\epsilon_{1}, \epsilon_{2}, \epsilon_{3}\right)$, where $\epsilon_{i}$ is either 1 or $-1, H(D F(X)) H$ has nonpositive off-diagonal elements for $X \in D$, where $D F(X)$ is the Jacobian of Eq. (4.1). It is shown in [22] that if $D$ is convex the flow of such a system preserves for $t<0$ the partial order in $R^{3}$ defined by the orthant

$$
K_{1}=\left\{\left(X_{1}, X_{2}, X_{3}\right) \in R^{3} \mid \epsilon_{i} X_{i} \geq 0\right\} .
$$

Hirsch [7] and Smith [22, 27] proved that three-dimensional competitive systems that live in convex sets have the Poincare-Bendixson property [15]; that is, any nonempty compact omega limit set that contains no equilibria must be a closed orbit. 
Lemma 5.1. Assume $D$ is convex and bounded. Suppose the system (5.1) is competitive and permanent and has the property of stability of periodic orbits. If $\bar{x}_{0}$ is the only equilibrium point in int $D$ and if it is locally asymptotically stable, then it is globally asymptotically stable in int $D$.

By looking at its Jacobian matrix and choosing the matrix $H$ as

$$
H=\left(\begin{array}{ccc}
1 & 0 & 0 \\
0 & -1 & 0 \\
0 & 0 & 1
\end{array}\right)
$$

we can see that the system (1.2) is competitive in $\Omega$, with respect to the partial order defined by the orthant $K_{1}=\left\{(T, I, V) \in R^{3}: T \geq 0, I \leq 0, V \geq 0\right\}$. Our main results will follow from this observation and the above theorems.

Theorem 5.2. If the conditions of Theorem 5.1 and Condition (iv): $d>\frac{a s \delta}{d}$ hold, then the positive equilibrium $E^{*}$ of the system (1.2) is globally asymptotically stable.

Proof. The system (1.2) is competitive, permanent if $R_{0}>1$, and the only equilibrium point $E^{*}$ of the system (1.2) is locally asymptotically stable if the conditions of Theorem 5.1 hold true. Furthermore, in accordance with Lemma 5.1 (where we can choose $D=\Omega$ ), Theorem 5.2 would be established if we show that the system (1.2) has the property of the stability of periodic orbits. In the following, we prove it.

Let $P(t)=(T(t), I(t), V(t))$ be a periodic solution whose orbit $\Gamma$ is contained in $\operatorname{int}\left(R_{+}^{3}\right)$, suppose that its minimal period is $\omega>0$. The second compound equation is following periodic linear system:

$$
Z^{\prime}(t)=\frac{\partial f^{[2]}}{\partial x}(P(t)) Z(t)
$$

where $Z=\left(Z_{1}, Z_{2}, Z_{3}\right)^{\top}$ and $\frac{\partial f}{\partial x}$ is derived from the Jacobian matrix of the system (1.2) and defined as follows:

$$
\frac{\partial f^{[2]}}{\partial x}=\left(\begin{array}{ccc}
-d-\delta-\frac{\beta V+b \beta V^{2}}{(1+a T+b V+a b T V)^{2}} & \frac{\beta T+b \beta T^{2}}{(1+a T+b V+a b T V)^{2}} & \frac{\beta T+b \beta T^{2}}{(1+a T+b V+a b T V)^{2}} \\
p & -d-c-\frac{\beta V+b \beta V^{2}}{(1+a T+b V+a b T V)^{2}} & 0 \\
0 & \frac{\beta V+b \beta V^{2}}{(1+a T+b V+a b T V)^{2}} & -\delta-c
\end{array}\right) .
$$

For the solution $P(t)$, the equation (5.2) becomes

$$
\left\{\begin{array}{l}
\dot{Z}_{1}(t)=\left(-d-\delta-\frac{\beta V+b \beta V^{2}}{(1+a T+b V+a b T V)^{2}}\right) Z_{1}+\frac{\beta T+b \beta T^{2}}{(1+a T+b V+a b T V)^{2}}\left(Z_{2}+Z_{3}\right), \\
\dot{Z}_{2}(t)=p Z_{1}+\left(-d-c-\frac{\beta V+b \beta V^{2}}{(1+a T+b V+a b T V)^{2}}\right) Z_{2}, \\
\dot{Z}_{3}(t)=\frac{\beta V+b \beta V^{2}}{(1+a T+b V+a b T V)^{2}} Z_{2}-(\delta+c) Z_{3} .
\end{array}\right.
$$

To prove that (5.3) is globally asymptotically stable, we shall use following Lyapunov function

$$
L\left(Z_{1}, Z_{2}, Z_{3} ; T(t), I(t), V(t)\right)=\sup \left\{\left|Z_{1}\right|, \frac{I}{V}\left(\left|Z_{2}\right|+\left|Z_{3}\right|\right)\right\} .
$$


The function (5.4) is positive, but not differentiable everywhere. Fortunately, this lack of differentiability can be remedied by using the right derivative of $L(t)$, denoted as $D_{+} L(t)$. Then we have the following equalities:

$$
\left\{\begin{array}{l}
\left|\dot{Z}_{1}(t)\right| \leq-\left(d+\delta+\frac{\beta V+b \beta V^{2}}{(1+a T+b V+a b T V)^{2}}\right)\left|Z_{1}\right|+\frac{\beta T+b \beta T^{2}}{(1+a T+b V+a b T V)^{2}}\left(\left|Z_{2}\right|+\left|Z_{3}\right|\right), \\
\left|\dot{Z}_{2}(t)\right| \leq p\left|Z_{1}\right|+\left(d+c+\frac{\beta V+b \beta V^{2}}{(1+a T+b V+a b T V)^{2}}\right)\left|Z_{2}\right| \\
\left|\dot{Z}_{3}(t)\right| \leq \frac{\beta V+b \beta V^{2}}{(1+a T+b V+a b T V)^{2}}\left|Z_{2}\right|-(\delta+c)\left|Z_{3}\right| .
\end{array}\right.
$$

Therefore,

$$
\begin{aligned}
D_{+} \frac{I}{V}\left|\dot{Z}_{2}(t)\right|+\left|\dot{Z}_{3}(t)\right| & =\left(\frac{\dot{I}}{I}-\frac{\dot{V}}{V}\right) \frac{I}{V}\left(\left|Z_{2}\right|+\left|Z_{3}\right|\right)+\frac{I}{V} D_{+}\left(\left|Z_{2}\right|+\left|Z_{3}\right|\right) \\
& \leq\left(\frac{I}{I}-\frac{V}{V}\right) \frac{I}{V}\left(\left|\dot{Z}_{2}\right|+\left|\dot{Z}_{3}\right|\right)+\frac{p I}{V}\left|Z_{1}\right| \\
& -(d+c) \frac{I}{V}\left|Z_{2}\right|-(c+\delta) \frac{I}{V}\left|Z_{3}\right| .
\end{aligned}
$$

Let $d_{1}=\min \{d+c, \delta+c\}$. Define

$$
\begin{gathered}
g_{1}(t)=-d-\delta-\frac{\beta V+b \beta V^{2}}{(1+a T+b V+a b T V)^{2}}+\frac{V}{I} \frac{\beta T+b \beta T^{2}}{(1+a T+b V+a b T V)^{2}}, \\
g_{2}(t)=p \frac{I}{V}+\left(\frac{\dot{I}}{I}-\frac{\dot{V}}{V}-c-d_{1}\right) \leq \frac{\dot{I}}{I}-d_{1} .
\end{gathered}
$$

From the last two equations of the system (1.2), we have

$$
\begin{aligned}
g_{1}(t) & \leq-d-\delta-\frac{\beta V+b \beta V^{2}}{(1+a T+b V+a b T V)^{2}}+\left(\frac{\dot{I}}{I}+\delta\right)+a\left(\frac{s}{d}+1\right)\left(\frac{\dot{I}}{I}+\delta\right) \\
& \leq-d+a \delta\left(\frac{s}{d}+1\right)+\left[1+a\left(\frac{s}{d}+1\right)\right] \frac{\dot{I}}{I} .
\end{aligned}
$$

Thus, we obtain

$$
D_{+} L(t) \leq \sup \left\{g_{1}(t), g_{2}(t)\right\} L(t) \text {. }
$$

From (5.6)-(5.8), we obtain $\sup \left\{g_{1}(t), g_{2}(t)\right\} \leq-\delta_{1}+\frac{\dot{I}}{I}$, where $\delta_{1}=\min \left\{d_{1}\right.$, $\left.d-a \delta\left(\frac{s}{d}+1\right)\right\}$.

Thus, we obtain

$$
\sup \left\{g_{1}(t), g_{2}(t)\right\} \leq\left[1+a\left(\frac{s}{d}+1\right)\right] \frac{\dot{I}}{I}-\delta_{1} .
$$

Hence,

$$
\int_{0}^{\omega} \max \left\{g_{1}(t), g_{2}(t)\right\} d t \leq\left.\left[1+a\left(\frac{s}{d}+1\right)\right] I(t)\right|_{0} ^{\omega}-\delta_{1} \omega=-\delta_{1} \omega,
$$

since $I(t)$ is periodic of minimal period $\omega$. From (5.8) and (5.9), we have $L(t) \rightarrow 0$ as $t \rightarrow \infty$. Therefore, $\left(Z_{1}(t), Z_{2}(t), Z_{3}(t)\right) \rightarrow(0,0,0)$ as $t \rightarrow \infty$.

This implies that the linear system Eq. (5.3) is asymptotically stable and therefore the periodic solution is asymptotically orbitally stable. This proves Theorem 5.2. 
TABLE 1. Parameter values used for simulation

\begin{tabular}{llll}
\hline Parameters & Range of the parameters & Source & Values \\
\hline$s$ (T-cells source term) & $0-10$ cells mm $^{-3} \mathrm{day}^{-1}$ & {$[4,8,17]$} & 10 \\
$d$ (Death rate of healthy T-cells) & $0.007-0.1 \mathrm{day}^{-1}$ & {$[17]$} & 0.09 \\
$\beta$ (Viral infectivity rate) & $0.00025-0.5$ virions & {$[4,8,17]$} & 0.0025 \\
$\delta$ (Death rate of infected T-cells) & $0.2-0.5 \mathrm{day}^{-1}$ & {$[4,5,20]$} & 0.4 \\
$c$ (Clearance rate of virus) & $2.4-3$ day $^{-1}$ & {$[4,17,20]$} & 2.4 \\
$p$ (Virion production rate) & $29-376 /$ day & {$[2,13]$} & 300 \\
$a$ (Positive parameters that describe & Assumed & & 0.0015 \\
$\quad$ the effects of capture rate) & & & 0.4 \\
$b$ (Positive parameters that describe & Assumed & & \\
$\quad$ the effects of capture rate) & & & \\
\hline
\end{tabular}

\section{Numerical simulation}

In this section, we perform some numeric simulations to demonstrate the theoretical results obtained in Section 5 by using Matlab 9.5. We present the numerical simulations to observe the dynamics of the system with a set of parameter values in Table 1 . We can get that the system (1.2) exists a unique positive equilibrium $E^{*}(104.9265344,1.113223809,139.1529762)$. We have seen in previous sections that the basic reproduction number $R_{0}$ plays a decisive rule in determining the virus dynamics. We can also get $R_{0}=59.52380952>1$. Moreover, the eigenvalues associated with the characteristic equation

$$
\lambda^{3}+2.994583397 \lambda^{2}+1.453113335 \lambda+0.1115940103=0
$$

are $-0.09468969487,-0.4887899513,-2.411103751$. Finally, we can see that the Condition (iv) of Theorem 5.2 is satisfied. Hence, the positive steady state $E^{*}$ is locally asymptotically stable (See Fig. 1).

However, Fig. 2 indicates that the disease steady state $E^{*}$ is globally asymptotically stable although the condition (iv) of Theorem 5.2 is not satisfied. Thus, we conjecture that the unique disease steady state $E^{*}$ is globally asymptotically stable only if the conditions of Theorem 5.1 are satisfied.

A model for HIV infection similar to (1.2) but using a simplified "massaction" term in which the rate of infection is given by $\beta T V$ has been proposed in $[18,19]$. The model and the global dynamics of the model (1.2), however, have not been rigorously established in the literature. The difference in the proliferation term does change the basic reproduction number. It will change the count of $\mathrm{CD}^{+}{ }^{+} \mathrm{T}$-cell at equilibrium level, and it also changes the equilibrium level of viral load during chronic infection. In Fig. 3 and Fig. 4, we present the different values of $b$ and $a$, respectively. We can find that the smaller $a$ (or larger $b$ ), the smaller $T$ and the higher $V$.

\section{Discussion}

In this paper, we investigate a differential equation model of HIV infection of $\mathrm{CD}^{+}$T-cells with Crowley-Martin functional response. By stability analysis 

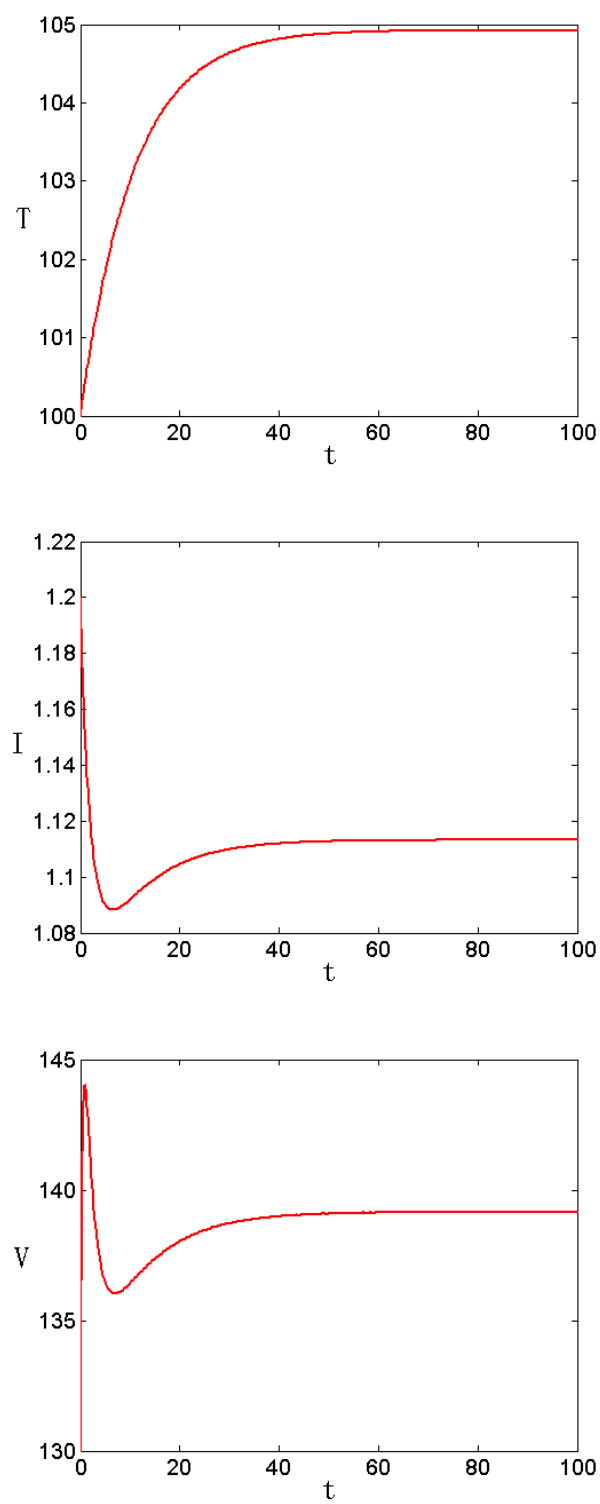

Fig.1: System (1.2) has one positive equilibrium $E^{*}$ and it is globally asymptotically stable if the conditions of Theorem 5.2 are satisfied. 

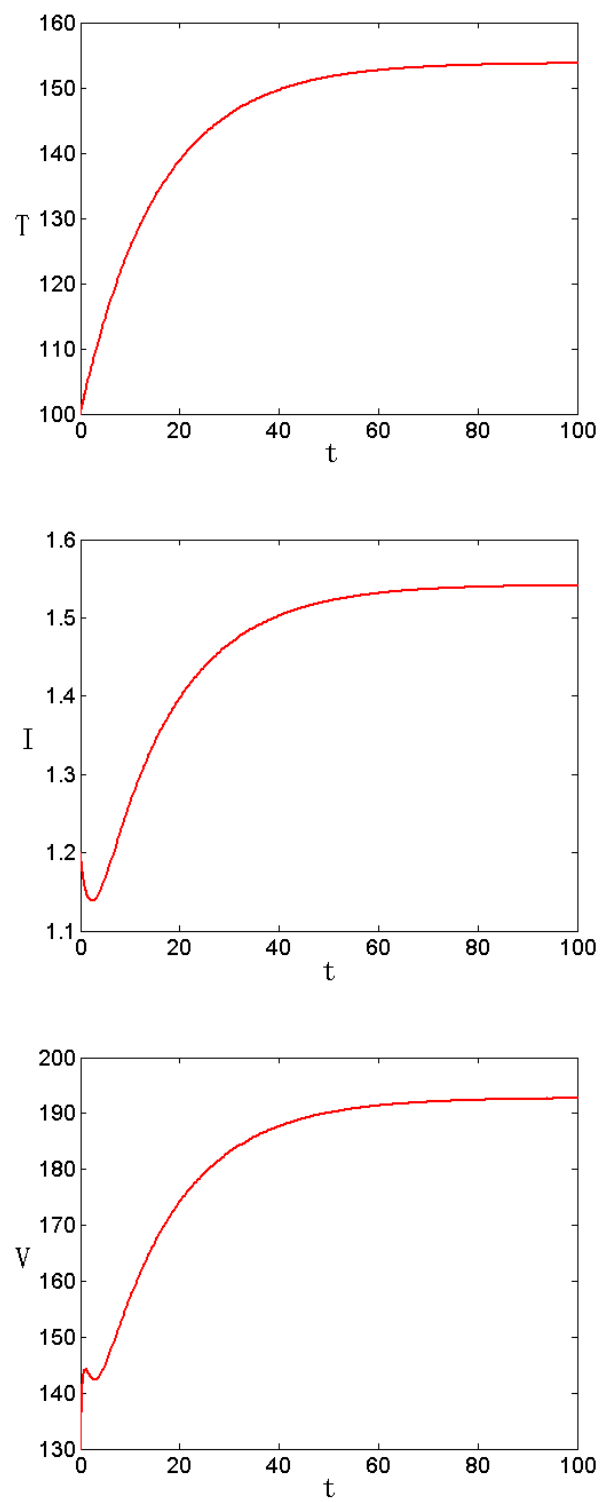

Fig.2: System (1.2) has one positive equilibrium $E^{*}$ and it is globally asymptotically stable if the condition (iv) of Theorem 5.2 are not satisfied. 

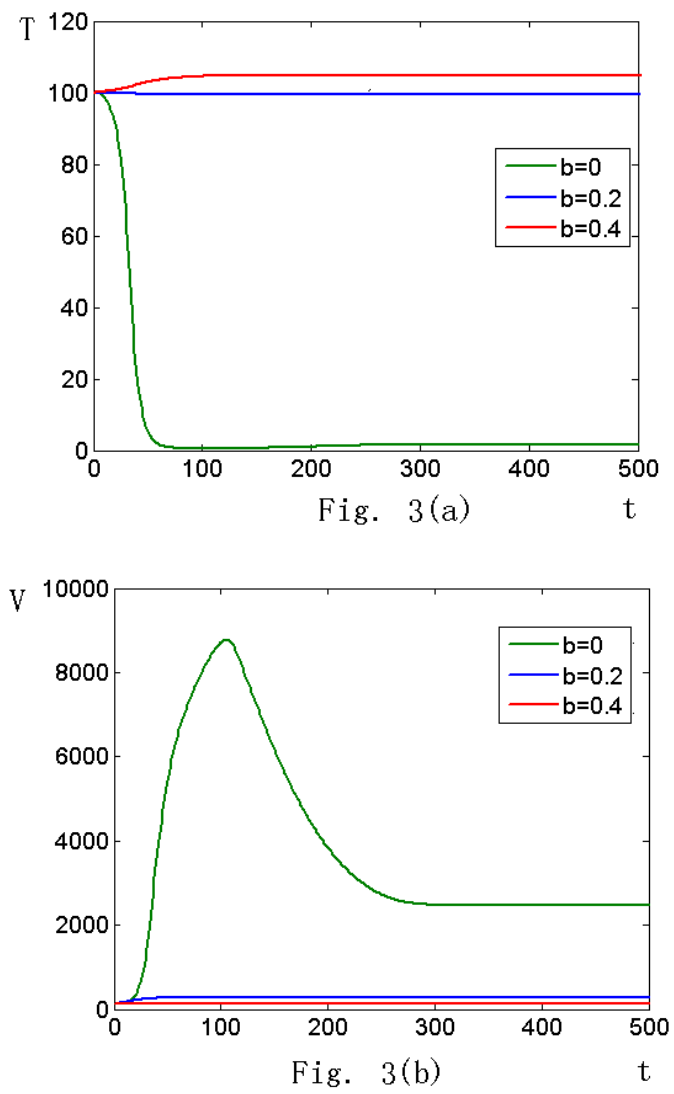

Fig.3: Variations of healthy $\mathrm{CD} 4^{+}$cells $(T)$ and virus population $(V)$ for different values $b$.

we obtain sufficient conditions on the parameters for the global stability of the infected steady state and the infection-free steady state.

If we compare model (1.2) with the basic model (1.1), we find that virus load decreases because of the difference in the infection rate. When $a=b=0$, the model (1.2) coincides with the basic model (1.1), of which unique infected steady state is globally asymptotically stable if $R_{0}^{*}=\frac{s p \beta}{\delta c d}>1$. If $a>0$, then $R_{0}^{*}>R_{0}$ and $\lim _{a \rightarrow 0} R_{0}=R_{0}^{*}$. Combination of numerical simulation, we can obtain that parameter $a$ is important to model (1.2). Although parameter $b$ is independent with $R_{0}$, it changes the count of $\mathrm{CD} 4^{+} \mathrm{T}$-cell at equilibrium level, and it also changes the equilibrium level of viral load during chronic infection. Hence, parameter $b$ is also important to model (1.2). That is to say, model (1.2) is more reasonable than model (1.1). We can monitor the values of $a$ and $b$ to help us solve some disease control problems of AIDS. 

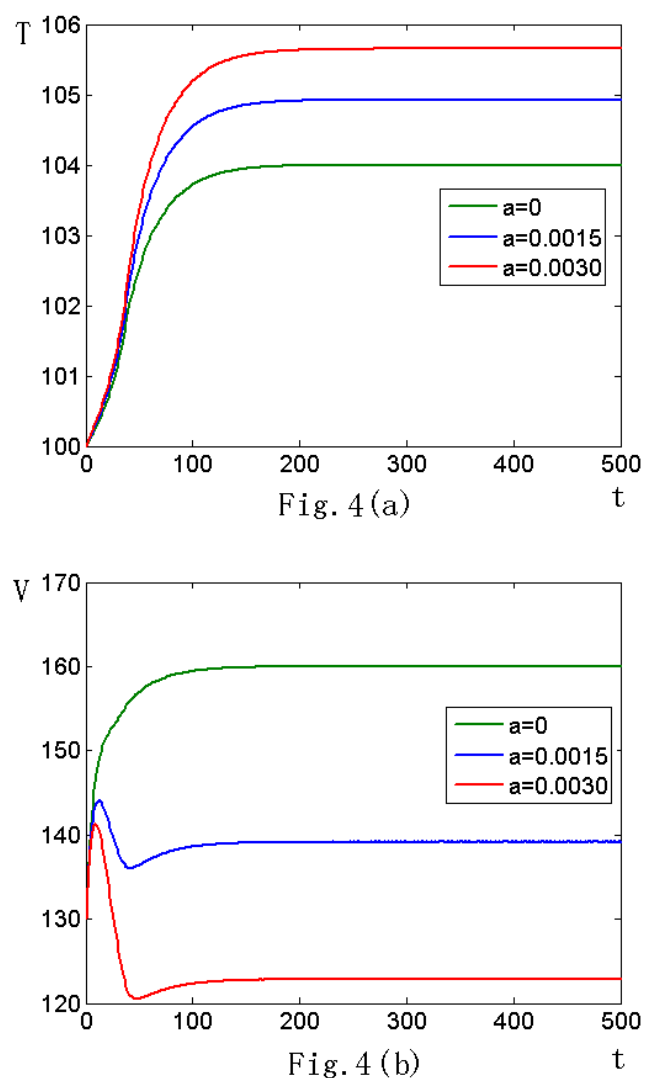

Fig.4: Variations of healthy $\mathrm{CD} 4^{+}$cells $(T)$ and virus population $(V)$ for different values $a$.

If we assume that the population dynamics of $\mathrm{CD} 4^{+}$cells is following $[25$, $26,19]$ :

$$
\frac{d T}{d t}=s+r T\left(1-\frac{T}{T_{\max }}\right)-d T,
$$

where $r$ is the maximum proliferation rate and $T_{\max }$ is the $T$ cell population density at which proliferation shuts off, the model (1.2) be modified as the following:

$$
\left\{\begin{array}{l}
\frac{d T}{d t}=s+r T\left(1-\frac{T}{T_{\max }}\right)-d T-\frac{\beta T V}{1+a T+b V+a b T V} \\
\frac{d I}{d t}=\frac{\beta T V}{1+a T+b V+a b T V}-\delta I \\
\frac{d V}{d t}=p I-c V .
\end{array}\right.
$$


Furthermore, we can consider "cure rate" [25] in the dynamics of (7.1). Hence, we can present the following model:

$$
\left\{\begin{array}{l}
\frac{d T}{d t}=s+r T\left(1-\frac{T}{T_{\max }}\right)-d T-\frac{\beta T V}{1+a T+b V+a b T V}+\rho I, \\
\frac{d I}{d t}=\frac{\beta T V}{1+a T+b V+a b T V}-\delta I-\rho I, \\
\frac{d V}{d t}=p I-c V,
\end{array}\right.
$$

where $\rho$ is the rate of "cure". We can also include "intracellular" delay [26]:

$$
\left\{\begin{aligned}
\frac{d T}{d t} & =s+r T\left(1-\frac{T}{T_{\max }}\right)-d T-\frac{\beta T V}{1+a T+b V+a b T V}+\rho I, \\
\frac{d I}{d t} & =\frac{\beta e^{-m} T(t-\tau) V(t-\tau)}{1+a T(t-\tau)+b V(t-\tau)+a b T(t-\tau) V(t-\tau)}-\delta I-\rho I, \\
\frac{d V}{d t} & =p I-c V
\end{aligned}\right.
$$

where the term $e^{-m \tau}$ accounts for cells that are infected at time $t$ but die before becoming productively infected $\tau$ time units later (i.e., if we assume a constant death rate $m$ for infected but not yet virus-producing cells, the probability of surviving from time $t-\tau$ to $t$ is just $e^{-m \tau}$ ).

From above, we can see the novelty of the model (1.2) compared to the models studied in [25] and [26] is modified the infection rate. And CrowleyMartin type of functional response is more appropriate than bilinear infection rate. The model (1.2) would be a positive role to the study of infectious diseases of AIDS.

Finally, models (7.1), (7.2) and (7.3) are more reasonable than model (1.2). And the dynamics behaviors of models (7.1), (7.2) and (7.3) are more complex than (1.2). We leave it in the future.

Acknowledgments. We are grateful to anonymous referees for their extremely insightful comments which improved our paper.

\section{References}

[1] S. Bonhoeffer, R. M. May, G. M. Shaw, and M. A. Nowak, Virus dynamics and drug therapy, Proc. Natl. Acad. Sci. USA 94 (1997), 6971-6976.

[2] S. M. Ciupe, R. M. Ribeiro, P. W. Nelson, and A. S. Perelson, Modeling the mechanisms of acute hepatitis $B$ virus infection, J. Theor. Biol. 247 (2007), no. 1, 23-35.

[3] P. H. Crowley and E. K. Martin, Functional responses and interference within and between year classes of a dragonfly population, J. North. Am. Benth. Soc. 8 (1989), 211-221.

[4] R. V. Culshaw and S. G. Ruan, A delay-differential equation model of HIV infection of CD4+ T-cells, Math. Biosci. 165 (2000), 27-39.

[5] R. V. Culshaw, S. G. Ruan, and R. J. Spiteri, Optimal HIV treatment by maximising immune response, J. Math. Biol. 48 (2004), no. 5, 545-562.

[6] F. R. Gantmacher, The Theory of Matrices, Chelsea Publ. Co., New York, 1959.

[7] M. W. Hirsch, Systems of differential equations which are competitive or cooperative $I V$, SIAM J. Math. Anal. 21 (1990), 1225-1234.

[8] P. De Leenheer and H. L. Smith, Virus dynamics: a global analysis, SIAM J. Appl. Math. 63 (2003), no. 4, 1313-1327. 
[9] D. Li and W. Ma, Asymptotic properties of a HIV-1 infection model with time delay, J. Math. Anal. Appl. 335 (2007), no. 1, 683-691.

[10] A. L. Lloyd, The dependence of viral parameter estimates on the assumed viral life cycle: limitations of studies of viral load data, Proc. R. Soc. Lond. B 268 (2001), 847-854.

[11] A. R. McLean and T. B. L. Kirkwood, A model of human immunodeficiency virus infection in T helper cell clones, J. Theor. Biol. 147 (1990), 177-203.

[12] A. R. McLean, M. M. Rosado, F. Agenes, R. Vasconcellos, and A. A. Freitas, Resource competition as a mechanism for $B$ cell homeostasis, Proc. Natl Acad. Sci. USA 94 (1997), 5792-5797.

[13] L. Q. Min, Y. M. Su, and Y. Kuang, Mathematical analysis of a basic virus infection model with application to HBV infection, Rocky Mountain J. Math. 38 (2008), no. 5, 1573-1585.

[14] J. E. Mittler, B. Sulzer, A. U. Neumann, and A. S. Perelson, Influence of delayed viral production on viral dynamics in HIV-1 infected patients, Math. Biosci. 152 (1998), $143-163$.

[15] J. S. Muldowney, Compound matrices and ordinary differential equations, Rocky Mountain J. Math. 20 (1990), no. 4, 857-872.

[16] N. Nagumo, Über die lage der integralkurven gewohnlicher differential gleichungen, Proc. Phys-Math. Soc. Japan 24 (1942), 551-559.

[17] P. W. Nelson, J. D. Murray, and A. S. Perelson, A model of HIV-1 pathogenesis that includes an intracellular delay, Math. Biosci. 163 (2000), no. 2, 201-215.

[18] A. S. Perelson, D. E. Kirschner, and R. de Boer, Dynamics of HIV infection of CD4+ T cells, Math. Biosci. 114 (1993), 81-125.

[19] A. S. Perelson and P. W. Nelson, Mathematical analysis of HIV-I dynamics in vivo, SIAM Rev. 41 (1999), 3-44.

[20] A. S. Perelson, A. U. Neumann, M. Markowitz, et al., HIV-1 dynamics in vivo: virion clearance rate, infected cell life-span, and viral generation time, Science 271 (1996), 1582-1586.

[21] H. L. Smith, Monotone dynamical systems: An Introduction to the theory of competitive and cooperative systems, Trans. Amer. Math. Soc., vol. 41, 1995.

[22] _ Systems of ordinary differential equations which generate an order preserving flow, SIAM Rev. 30 (1998), 87-98.

[23] X. Y. Song and A. U. Neumann, Global stability and periodic solution of the viral dynamics, J. Math. Anal. Appl. 329 (2007), no. 1, 281-297.

[24] H. R. Thieme, Persistence under relaxed point-dissipativity (with applications to an endemic model), SIAMJ. Math. Anal. 24 (1993), 407-435.

[25] X. Y. Zhou, X. Y. Song, and X. Y. Shi, A differential equation model of HIV infection of CD4 ${ }^{+}$CD4 + T-cells with cure rate, J. Math. Anal. Appl. 342 (2008), no. 2, 1342-1355.

[26] Analysis of stability and Hopf bifurcation for an HIV infection model with time delay, Appl. Math. Comput. 199 (2008), no. 1, 23-38.

[27] H. R. Zhu and H. L. Smith, Stable periodic orbits for a class of three-dimensional competitive systems, J. Differential Equations 110 (1994), no. 1, 143-156.

XUEYONG ZHOU

School of Mathematical Sciences

Nanjing Normal University

Nanjing 210046, Jiangsu, P. R. China

AND

College of Mathematics and Information Science

Xinyang Normal University

Xinyang 464000, Henan, P. R. China

E-mail address: xueyongzhou@126.com 
JiNGAN CUI

SCHOOL OF SCIENCE

Beijing University of Civil Engineering and Architecture

BeiJing 100044, P. R. China

E-mail address: cuijingan@bucea.edu.cn 